\title{
An Evidence-Based Review of Fremanezumab for the Treatment of Migraine
}

\author{
Ivan Urits · Gavin Clark · Daniel An · Bredan Wesp · Rebecca Zhou • \\ Ariunzaya Amgalan · Amnon A. Berger · Hisham Kassem • \\ Anh L. Ngo • Alan D. Kaye - Rachel J. Kaye · Elyse M. Cornett • \\ Omar Viswanath
}

Received: February 5, 2020 / Published online: March 28, 2020

(c) The Author(s) 2020

\begin{abstract}
Migraine headache is a common, chronic, debilitating disease with a complex etiology. Current therapy for migraine headache comprises either treatments targeting acute migraine pain or prophylactic therapy aimed at increasing the length of time between migraine episodes. Recent evidence suggests that calcium gene-related peptide (CGRP) is a critical component in the pathogenesis of migraines. Fremanezumab, a monoclonal antibody against CGRP, was recently approved by the Food and
\end{abstract}

Digital Features To view enhanced digital features for this article go to https://doi.org/10.6084/m9.figshare. 11890503.

I. Urits $(\bowtie) \cdot$ A. A. Berger

Beth Israel Deaconess Medical Center, Department of Anesthesiology, Critical Care, and Pain Medicine, Harvard Medical School, Boston, MA, USA

e-mail: iurits@bidmc.harvard.edu

G. Clark · D. An · B. Wesp · R. Zhou · A. Amgalan Georgetown University School of Medicine,

Washington, DC, USA

H. Kassem

Department of Anesthesiology, Mount Sinai

Medical Center, Miami Beach, FL, USA

\section{A. L. Ngo}

Department of Anesthesiology, Perioperative and Pain Medicine, Brigham and Women's Hospital, Boston, MA, USA
Drug Administration (FDA) after multiple studies showed that it was well-tolerated, safe, and effective in the treatment of migraines. Further research is needed to elucidate the long-term effects of fremanezumab and CGRP-antagonists in general, and additional data is required in less healthy patients to estimate its effects in these populations and potentially increase the eligible group of recipients. This is a comprehensive review of the current literature on the efficacy and safety of fremanezumab for the treatment of chronic migraine. In this review we provide an update on the epidemiology, pathogenesis, diagnosis, and current treatment of migraine, and summarize the evidence for fremanezumab as a treatment for migraine.

\author{
A. L. Ngo \\ Department of Pain Medicine, Pain Specialty Group, \\ Newington, NH, USA \\ A. D. Kaye · E. M. Cornett \\ Department of Anesthesiology, Louisiana State \\ University Health Shreveport, Shreveport, LA, USA \\ R. J. Kaye \\ Medical University of South Carolina, Charleston, \\ SC, USA \\ O. Viswanath \\ Valley Anesthesiology and Pain Consultants - \\ Envision Physician Services, Phoenix, AZ, USA
}


Keywords: Chronic Fremanezumab; Headache; Monoclonal antibody

\section{Key Summary Points}

Migraine headache is a common, chronic, debilitating disease with a complex etiology.

Recent evidence suggests that calcium gene-related peptide (CGRP) is a critical component in the pathogenesis of migraines.

Fremanezumab, a monoclonal antibody against CGRP, has been recently approved by the FDA after multiple studies showed that it was well-tolerated, safe, and effective in the treatment of migraines.

Long-term studies are required to monitor the long-term effects and financial effectiveness of this therapy.

\section{INTRODUCTION}

Migraine, as defined by the International Headache Society's International Classification of Headache Disorders 3 criteria (ICHD-3), is a recurrent and debilitating primary headache disorder, with individual attacks lasting from 4 to $72 \mathrm{~h}$ that impact and/or are exacerbated by regular physical activity such as walking [1]. The headaches are most commonly described as pulsatile in nature and localized unilaterally, and may or may not be accompanied by prodromal, aura, and resolution symptoms [1, 2]. The classification of migraine remains relatively

\footnotetext{
O. Viswanath

Department of Anesthesiology, Creighton

University School of Medicine, Omaha, NE, USA

O. Viswanath

Department of Anesthesiology, University of

Arizona College of Medicine-Phoenix, Phoenix, AZ, USA
}

broad, as the specifics of each attack can vary considerably between individuals and even within the same individual [3]. Nausea, photophobia, and phonophobia are symptoms commonly associated with migraines [1]. Aura symptoms present as the gradual development of unilateral visual, motor, or sensory deficits, among others, that are fully-reversible and can continue into the onset of the headache itself [1]. Resolution symptoms are most commonly described as increased tiredness, asthenia, and increased somnolence [2]. The ICHD-3 further recognizes the frequency of attacks as a crucial element in migraine classification and has defined two categories, episodic migraine (EM) and chronic migraine $(\mathrm{CM})$; chronic migraine was previously referred to as transformed migraine. To be classified as $\mathrm{CM}$, headache attacks must occur $\geq 15$ days per month for 3 or more months; anything below this cutoff is therefore defined as EM [1]. Progression from EM to CM has been described and appears to occur at an annual rate of between 2.5 and 3\% in a process termed migraine chronification $[4,5]$.

This is a comprehensive review of the current literature on the efficacy and safety of fremanezumab for the treatment of chronic migraine. In this review we provide an update on the epidemiology, pathogenesis, diagnosis, and current treatment of migraine, and summarize the evidence for fremanezumab as a treatment for migraine. This article is based on previously conducted studies and does not contain any studies with human participants or animals performed by any of the authors.

\section{EPIDEMIOLOGY}

Migraine headaches are a relatively common and disabling global disease. Analysis of the Global Burden of Disease, Injuries, and Risk Factors Study from 2016 (GBD 2016) found the global total of migraineurs to have reached 1.04 billion, making migraine the sixth most prevalent cause of disease in that year [6]. On a more local scale, the 2004 American Migraine Prevalence and Prevention Study (AMPP) provided a longitudinal pool of information 
regarding the disease in the United States. In particular, analysis of the AMPP has estimated the U.S. prevalence of "severe headache" to be $17.4 \%$ (28,261 total people), approximately two thirds of which met the ICHD- 2 criteria for a migraine diagnosis [7]. Based on an earlier sampling of 20,000 households in the American Migraine Study II, just under a quarter of households contained at least one migraineur. This same survey data inquired after the impact of migraine on productivity. They found that close to one third of respondents missed 1 day of work or school in the last 3 months because of migraine, and more than half felt their work/ school productivity was significantly reduced [8]. Moreover, the disability impact of migraine appears to be increasing. Further study of the GBD 2015 found a statistically significant increase of $15.3 \%$ in all-age migraine disabilityadjusted life-years (DALYs) from 2005 to 2015 [9]. Another analysis of the GBD 2015 estimated that migraines are the second largest contributor $(13.1 \%)$ to all neurologic DALYs, behind stroke [10]. The distribution of disease does not appear to be evenly split between the sexes; it also appears to fade with age. There has been consistent evidence over time demonstrating that women are affected at rates between two and three times higher than men [7, 10-13]. Across all genders, migraine rates appear to peak anywhere between 25 and 50 years of age, and then proceed to decline in persons over 60 years old $[7,8,10,13]$.

\section{RISK FACTORS FOR MIGRAINE}

There is a dearth of available information regarding the risk factors associated with the development of initial EM. Progression of EM to $\mathrm{CM}$ has been studied heavily, with studies revealing the risk factors involved. The major factors indicated are age, female gender, comorbid depression, low socioeconomic status, baseline headache frequency, medication overuse, obesity, and caffeine use. These factors can be separated into those that are modifiable and those that are realistically unmodifiable.

\section{Non-Modifiable Risk Factors}

Migraine prevalence exhibits an age-related pattern of distribution, with a peak in prevalence between 25 and 50 years of age for all genders $[7,8,10,13]$. There is repeated evidence that the prevalence of migraine declines with advancing age, with the exception of females within the hormonally fluctuating period leading up to menopause, but this effect appears to diminish with eventual hormone stabilization [7]. Premenopausal women are also at a $75 \%$ increased risk of migraine in the 5 days surrounding ( 2 days before and 3 days after) the start of menstruation when compared with other points in their cycle [14]. Another factor that appears to be associated with increased chronicity is the presence of comorbid depression in those with EM, with the increased risk proportional to the severity of the depression $[4,15,16]$. Lastly, a significant increase in prevalence has been found related to lower socioeconomic status [13].

\section{Modifiable Risk Factors}

There are multiple sites of evidence linking an increase in baseline EM attack frequency with an exponential risk of progression to $\mathrm{CM}$ $[4,5,17]$. This is believed to be the result of increased central sensitization over time [17]. Medication overuse has also been identified as a predominant risk factor, but there is evidence that this depends on the medication. With acetaminophen as the control, both barbiturate and opiate overuse was linked to increased odds of development of CM [5]. In the AMPP study, monotherapy of triptans for treatment of EM was associated with increased risk of progression to CM. The same study found that nonsteroidal anti-inflammatory drugs (NSAIDs) use in migraineurs with a frequency of headache $>10$ days/month showed a dose-dependent increase in the risk of progression to CM. However, dual therapy with both triptans and NSAIDs was not associated with increased risk [18]. Obesity is another repeatedly linked risk factor. Individuals with a BMI $\geq 30$ experienced a greater frequency of migraine than 
those below that cutoff. The obese and morbidly obese BMI was also linked to greater severity of each headache attack, with the morbidly obese noting twice the headache pain of those with a normal BMI [4]. Lastly, individuals with $\mathrm{CM}$ were more likely to have been labeled as high caffeine consumers (defined as being in the upper quartile of caffeine consumption) before the onset of $\mathrm{CM}$ when compared with EM controls [19].

\section{DIAGNOSIS AND CLINICAL PRESENTATION OF MIGRAINE}

As the diagnosis of migraine requires the patient to recall the frequency, quality, and duration of their headaches, it is often helpful for the patient to maintain a headache diary for at least 1 month, in the hope of eliminating recall error. The diagnosis of migraine utilizes the International Headache Society's International Classification of Headache Disorders 3rd edition. The ICHD-3 splits EM into two types, with aura and without aura. The following is adapted from the ICHD-3 criteria [1].

\section{Migraine without Aura}

The patient must have had a minimum of five headaches lasting between 4 and $72 \mathrm{~h}$. The headache itself must also fulfill at least two of the following criteria: unilaterally located, pulsatile nature, moderate to severe pain, aggravated by or causing avoidance of regular daily activities. Throughout the ictal phase, the migraineur must also experience either nausea and/or vomiting or photophobia with phonophobia.

\section{Migraine with Aura}

The patient must have experienced two attacks that include fully reversible symptoms involving at least one of the following: visual, sensory, speech/language, motor, brainstem, or retinal disruption. These headaches must also have a minimum of three of the following: aura spreading gradually over $5 \mathrm{~min}$, succession of two or more aura symptoms, presence of an aura symptom lasting $5 \mathrm{~min}$ to $1 \mathrm{~h}$, a unilateral aura symptom, at least one aura is a positive symptom, the aura is combined with or followed by a headache within $1 \mathrm{~h}$.

\section{Chronic Migraine}

Chronic migraine can occur with or without aura but is principally defined by the increased regularity of migraine attacks. CM is classified as headache on $\geq 15$ days/month. This headache frequency must be maintained for more than 3 months. CM diagnosis is also indicated by relief of migraine by triptan or ergot derivative.

\section{Premonitory Symptoms}

Multiple studies have elucidated premonitory symptoms associated with migraine that can be used to identify the onset of migraine $[2,20]$. These symptoms can preempt the ictal phase of migraine by up to $72 \mathrm{~h}$, but predictive value is most accurate within $24 \mathrm{~h}$ [2]. Premonitory symptoms are often consistent between attacks within a single patient, and treatment of these symptoms appeared to reduce the severity of the oncoming migraine [2]. Given the relatively large window of time that these symptoms can present before onset of migraine and the potential benefit of early recognition and treatment, it may prove clinically relevant to note them. The most frequent premonitory symptoms were asthenia, difficulty concentrating, stiff neck, mood changes, gastrointestinal symptoms, and increased somnolence $[2,20,21]$.

Diagnosis of $\mathrm{CM}$ excludes the diagnosis of chronic tension-type headache, and is an important distinction, since misdiagnosis can result in inappropriate management [22]. Migraineurs with CM can have predominantly tension-type headache attacks but should still be diagnosed with $\mathrm{CM}$ if they have migraine headaches 8 days or more per month [22]. Chronic tension-type headaches present normally with bilateral distribution and lack the pulsatile quality and nausea, phonophobia, and photophobia of migraine headaches [22]. 


\section{PATHOPHYSIOLOGY OF MIGRAINE HEADACHES}

Although the mechanism of migraine has yet to be fully understood, current research has attempted to elucidate this disease process. Migraine is best understood as a neurovascular disorder originating from the brain and spreading to the trigeminal vascular system (TVS), provoking pain symptomology [23-27]. There are four phases of migraine: premonitory phase, aura phase, headache phase, and postdrome phase; these generally occur sequentially but can also show overlap and slight differences [28]. The premonitory phase involves a collection of sensory alterations, mood changes, polyuria, and irritability that can occur hours before the headache phase of migraine [28]. Functional brain imaging of migraineurs reveals activation of the brainstem and hypothalamus, leading to a hypothesis of dysfunctional brain activity in these areas $[29,30]$. More importantly, the alteration of afferent signals by the brainstem and hypothalamus can cause neurological symptoms such as photophobia and phonophobia seen in the premonitory phase, pointing to the brain as the principal issue in migraine $[20,21,29,31]$. About $15 \%$ of migraineurs experience aura, which describes transient focal neurologic symptoms that result in visual deficits immediately before the headache phase [23]. A growing body of evidence indicates that migraine aura is closely related to cortical spreading depression (CSD), a slow propagating wave along the brain surface involving neurons, glial cells, and vasculature, as originally suggested by Leao's animal model experiments $[32,33]$. This wave consists of an initial depolarization in areas of the occipital lobe followed by impaired neuronal activity and spreading oligemia that can lead to the clinical features of scintillating scotoma seen in migraine aura [32, 34]. In further support of this association, functional MRI in humans during migraine aura attacks reveals similar patterns of CSD in the visual cortex [35]. In the literature, it is widely accepted that CGRP and the TVS play major roles in the pathogenesis of migraine [36]. The TVS includes the nucleus caudalis, trigeminal ganglia, and ophthalmic division of trigeminal nerve, together conveying nociceptive sensory information from blood vessels in the meninges to higher-order neurons [37]. Activation of trigeminal nerves causes the release of several neuropeptides including CGRP, substance $P$, and pituitary adenylate cyclase-activating polypeptide-38 (PACAP-38) [38-41]. CGRP is the most potent vasodilator and is known to cause migraine attacks via its release in the cerebral vascular beds [36]. Recent studies suggest that CSD initiates activation of the TVS, and this CSD-mediated CGRP release coincides with the onset of headache due to activation of meningeal nociceptors on nerve endings of peripheral trigeminal nerves [42]. Additionally, CGRP is implicated in neurogenic inflammation resulting in hyperresponsive stimulation of primary nociceptive trigeminal fibers [43]. This unregulated activation leads to sensitization of successive pain transmission neurons within the central nervous system (CNS) $[43,44]$. The peripheral sensitization decreases firing thresholds of nociceptive fibers and increases the magnitude of response to nociceptive stimulus, leading to hyperalgesia and allodynia [43, 45]. The peripheral and central pain sensitization due to the abnormal activation of the TVS is causative of sustained headache [46].

\section{CURRENT TREATMENT}

\section{Acute Migraine Headache Therapy}

Current acute migraine therapies include nonspecific analgesics such as NSAIDs and specific drugs such as the ergots and triptans aimed at aborting migraine attacks [47]. Among these, triptans have the best evidence for effectiveness as acute migraine therapy and are frequently prescribed [37, 48]. Triptans such as sumatriptan are selective 5-hydroxytryptamine (5HT1B/ 1D) receptor agonists that act as potent vasoconstrictors by opposing the vasodilatory effects in cerebral vasculature and inhibiting neurotransmitter release in trigeminal nociceptive neurons [47]. It is important to note that administration of acute therapy before the 
onset of migraine attacks is more effective than after migraine symptoms emerge [49]. The use of triptans is limited to acute therapy, since the risk of developing medication overuse headache $(\mathrm{MOH})$ correlates with frequency of triptan use [50]. Additionally, triptans are contraindicated in patients with cardiovascular risk, due to undesirable vasoconstrictive effects $[37,51,52]$.

\section{Migraine Headache Preventive Therapy}

Unlike the drugs used for acute migraine therapy, migraine preventive therapy aims to reduce the incidence of migraine attacks. Hence, these types of treatments are more suitable for EM and CM with frequent recurrent attacks [50]. These preventive medications were originally designed for other medical conditions including depression, epilepsy, and hypertension before being used for migraine therapy [46].

\section{Onabotulinumtoxin A}

Onabotulinumtoxin A (OBT-A) is a locally injectable drug approved for prophylaxis of $\mathrm{CM}$ and is used to reduce migraine attacks and improve patients' pain profiles [53-56]. OBT-A has a long duration of action, allowing a 3-month drug delivery interval, and has a safe and tolerable drug profile, as shown in the PREEMPT study [53, 57]. It is thought that injection of OBT-A inhibits the release of many neurotransmitters including CGRP, substance $P$, and glutamate from peripheral trigeminal nociceptive neurons [58-60]. Currently, OBT-A is the only medicine approved by FDA for the prevention and treatment of CM [58].

\section{Topiramate}

Topiramate was originally approved by the FDA as an antiepileptic drug, but has also been found to be an effective prophylactic medication for EM and CM [61]. Topiramate involves voltagegated $\mathrm{Ca}^{2+}$ channels and GABA-mediated neuro-inhibition to raise neural thresholds and prevent the initiation of migraine attacks and CSD $[62,63]$. Common side effects of topiramate include weight loss, paresthesia, fatigue, and poor concentration, all making topiramate a less persuasive treatment option than OBT-A [64].

\section{Ditans}

Ditans, such as lasmiditan, have been developed as novel 5-HT1F receptor agonists and are currently undergoing clinical trials for acute migraine therapy [65]. 5-HT1F receptors are found within trigeminal nerve endings, and their activation is linked to the inhibition of CGRP release, without the vasoconstrictive characteristics observed with triptans [66]. The absence of vasoconstrictive characteristics makes ditan a promising new acute therapy option for patients with cardiovascular contraindications $[67,68]$.

\section{Gepants}

The importance of CGRP in the modulation of pain pathways in the brain has been gaining attention, and has led to pharmacological development of CGRP-targeted therapies such as gepants [69]. Three new gepants, namely rimegepant, ubrogepant, and atogepant, are currently undergoing phase 3 clinical trials and have shown positive efficacy and side effect profiles [70]. Gepants are small molecule CGRP receptor antagonists developed for acute migraine therapy [65]. In comparison with the vasoconstrictive adverse events (AEs) in triptans, recent studies show that gepants cause no vasoconstriction and are thus a promising addition to acute migraine therapies [71].

\section{Monoclonal CGRP Antibodies}

Another class of drugs that has been recently introduced is the group of monoclonal antibodies against CGRP or CGRP receptor for the prevention of EM and CM [72]. Recent clinical trials show very promising data indicating that monoclonal antibodies such as fremanezumab have high specificity for its target, long half-life, and promising safety and toxicity profiles $[73$, 74]. Fremanezumab, eptinezumab, galcanezumab, and erenumab are the first effective 
antibody treatments developed specifically for migraine prevention and need further investigation [75].

\section{FREMANEZUMAB}

Fremanezumab $\left(\right.$ Ajovy $\left.^{\circledR}\right)$ is a fully humanized IgG2 monoclonal antibody, approved by the FDA and the European Medicines Agency (EMA) for the prevention of episodic or chronic migraine in adults $[76,77]$. The drug was produced by Teva Pharmaceuticals via recombinant DNA technology in Chinese hamster ovary (CHO) cells [76]. Fremanezumab selectively binds to and blocks the calcitonin gene-related peptide (CGRP), a neuropeptide that is increased in migraine [76-79]. The recommended dosage is $225 \mathrm{mg}$ monthly or $675 \mathrm{mg}$ (three consecutive injections of $225 \mathrm{mg}$ each) every 3 months administered subcutaneously in the abdomen, thigh, or upper arm [76]. Each syringe delivers $225 \mathrm{mg}$ of the drug per $1.5 \mathrm{~mL}$ [76]. For multiple injections, the injection needs to be at a different location from the previous injection site but can be in the same general body area [76]. Fremanezumab needs to be stored in a refrigerator protected from light and should be discarded if it has been at room temperature for $24 \mathrm{~h}$ or longer [76]. Randomized placebo-controlled trials in adults with episodic and chronic migraine validated the effectiveness of fremanezumab as preventive therapy for migraine $[76,77,80-85]$. The most common adverse reactions reported in clinical trials were injection site reactions which include pain, induration, and erythema at the site of injection [76].

\section{CGRP AS A TARGET: MECHANISM OF ACTION}

The pathophysiology of migraine is linked to activation of the trigeminovascular system, which releases several neuropeptides including CGRP, a 37-amino acid peptide neurotransmitter found in sensory nerves throughout the central and peripheral nervous systems $[36,77-79,86-88]$. CGRP exists in two isoforms, $\alpha$ and $\beta$, but since the $\beta$ form is found in the enteric nervous system, hereafter the paper will focus on $\alpha$-CGRP, which is a peptide derived from the calcitonin gene on chromosome 11 via alternative mRNA splicing and proteolytic cleavage $[75,79,89,90]$. Since its discovery in 1982, CGRP has been found to play a critical role in the mechanism of migraine pathology $[79,91,92]$. Among the earliest evidence of the role of CGRP in migraine was the discovery by Goadsby et al., whose study was the first to show that during an acute migraine attack, the only neuropeptide that is released is CGRP $[79,93]$. Later clinical studies aligned with these findings and contributed additional evidence of elevated levels of CGRP in migraine [94-96]. Some clinical studies also provided evidence for a causal role of CGRP in migraine [97-99]. In response to cerebrovascular vasoconstriction, CGRP is released from vesicles in the trigeminal ganglion via calcium-dependent exocytosis and binds to the CGRP receptor, a heterodimer of calcitonin receptor-like receptor (CLR) and receptor activity-modifying protein 1 (RAMP1), to exert its potent vasodilatory effects $[36,79,90,100]$. The CGRP receptor complex is coupled to $G \alpha$ so that, when stimulated, it activates intracellular cAMP, amplifying protein kinase $\mathrm{A}$, which then phosphorylates multiple downstream targets [79, 90]. CGRP signaling ultimately results in vasodilation of intracranial blood vessels, contributing to the neurogenic inflammation that triggers migraine nociception $[79,87]$.

The monoclonal antibody fremanezumab targets the CGRP ligand, but the exact site of action is not fully understood [36]. It is thought that monoclonal antibodies act peripherally because they do not cross the blood-brain barrier [79, 87]. Possible sites of action for fremanezumab are the trigeminal ganglion and meningeal nociceptors $[78,87,101]$. A study in rats found that fremanezumab selectively inhibits activation of peripheral lightly myelinated

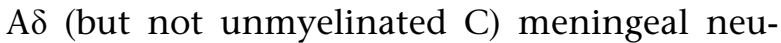
rons and central high-threshold (HT) [but not wide-dynamic-range (WDR)] trigeminovascular neurons by cortical spreading depression (CSD) in the meningeal sensory pathway [78, 87]. Based on their findings, the authors concluded 
that activation of $\mathrm{A} \delta$ meningeal pain fibers leads to activation of HT trigeminal neurons centrally, and then their axonal projections convey nociceptive signals to upstream CNS regions, resulting in the perception of migraine symptoms [78, 87]. This coincides with earlier immunohistochemical and in situ hybridization studies which showed that around half of the neurons in the trigeminal ganglion express CGRP, and that within the trigeminal ganglion/ nerve, CGRP is expressed in unmyelinated C sensory neurons/fibers, while CGRP receptors are found on myelinated A $\delta$ sensory neurons/fibers and Schwann cells [79, 91, 102-105]. Given that the trigeminovascular pathway activation is central to the transmission of migraine, blocking CGRP signaling with monoclonal antibodies such as fremanezumab effectively inhibits the trigeminovascular pain pathway, thus preventing migraine.

\section{PHARMACOKINETICS AND PHARMACODYNAMICS}

Fremanezumab specifically targets CGRP and prevents it from binding to its receptor, thereby preventing activation of the trigeminovascular pain pathway [36, 77-79, 86-88]. The pharmacological properties of fremanezumab make it a safe and tolerable drug for migraine prophylaxis. A single subcutaneous (SC) injection (225 mg, $675 \mathrm{mg}$, or $900 \mathrm{mg}$ ) took a median of 5-7 days to reach maximum concentration $\left(t_{\max }\right)[76,80,90]$. Dosing regimens of $225 \mathrm{mg}$ SC monthly and $675 \mathrm{mg}$ SC quarterly took about 168 days ( $\sim 6$ months) to reach steady state $[76,80]$. Like other monoclonal antibodies, fremanezumab is degraded into small peptides and amino acids by enzymatic proteolysis, not by cytochrome P450 enzymes, so it does not generate toxic metabolites [76]. Thus, the risk of hepatotoxicity or drug-drug interactions is low. Given that fremanezumab is a peptide, it must be administered parenterally and cannot easily cross membranes, resulting in a relatively small distribution volume of approximately 6 L [76]. Although an injection may not be the preferred form of drug administration, fremanezumab has a long half-life of approximately 31 days, which allows for less frequent injections (only once a month or once every 3 months) and may thus increase drug compliance [76]. A population analysis of 2287 subjects found no effect of age, race, sex, or weight on fremanezumab pharmacokinetics [76]. Also, administration of concomitant migraine medications such as analgesics, ergots, or triptans was not found to have any negative effects on fremanezumab efficacy $[76,80]$. No studies were conducted to determine the effect of renal impairment, severe hepatic impairment, or carcinogenesis on fremanezumab pharmacokinetics [76]. In a pooled safety analysis of four placebo-controlled phase $2 \mathrm{~b} / 3$ studies (total $n=2563$ ), the most common AEs were injection site reactions, most of which were mild to moderate [80, 85]. Serious AEs such as cardiovascular effects, hepatotoxicity, or hypersensitivity were rare and occurred at rates similar to those in placebo groups [80, 85]. Consistent with these findings, a year-long study of fremanezumab in adults with episodic and chronic migraine demonstrated that the efficacy and safety of the drug were maintained over 12 months of treatment [85, 106], but additional longitudinal studies are needed to confirm this. Fremanezumab is currently approved for use in adults, but it is important to note that there is insufficient data on safety in pregnant women, although studies in mating/ pregnant rats and rabbits found no adverse effects on fertility or embryo-fetal and postnatal development [76].

Melo-Carrillo et al. [78] conducted experiments investigating the effects of fremanezumab on neuronal activity in rats to better understand its role in reducing migraine frequency [78]. In the literature, CGRP has been implicated in the pathophysiology of migraine. Single-unit recording in the spinal trigeminal nucleus was performed. Central trigeminovascular neurons were then identified and labeled as WDR if the neuron showed incremental response to brush, pressure, and pinch, or HT if the neurons failed to respond to brush stimuli. Cortical spreading depression (CSD) was induced, and cortical activity was recorded using electrocorticography. Next, male and female rats were administered either IV fremanezumab at $30 \mathrm{mg} / \mathrm{kg}$ or a control (human 
IgG isotype antibody), and both spontaneous and evoked activity were measured in naive or CSD-sensitized neurons. The study found that fremanezumab selectively inhibited HT neurons and spared WDR neurons in the intracranial dura, and prevented CSD-induced activation and sensitization of HT neurons in male and female rats. Fremanezumab also decreased the sensitivity to dural stimulation, but not cutaneous facial or corneal stimulation. After administration of the control antibody, there was no change in sensitivity to dural stimulation in HT and WDR neurons, and there was sensitization of HT neurons after CSD induction. Given these findings, it appears that HT neurons play a significant role in the pathophysiology of migraine, allodynia, and central sensitization.

\section{Clinical Studies: Safety and Efficacy}

Bigal et al. [81] conducted a phase $2 \mathrm{~b}$ multicenter randomized controlled trial (RCT) on the safety and efficacy of TEV-48125 (fremanezumab) in the prevention of high-frequency episodic migraines (HFEM) [82]. The study included 297 individuals in the US, aged 18-65, who experienced migraine headaches 8-14 days per month and were otherwise healthy. They were randomized in a 1:1:1 fashion to three 28-day cycles. Each cycle consisted of three injections of either placebo, $225 \mathrm{mg}$ fremanezumab (one $225 \mathrm{mg}$, two placebo injections), or $675 \mathrm{mg}$ fremanezumab (three $225 \mathrm{mg}$ injections). Participants were permitted to be taking a stable dose of one standard migraine prevention medication for at least 2 months and to use an acute migraine medication up to 14 days per month prior to the study. During the treatment phase of the study, participants were allowed to use one concurrent migraine preventive medication, and to treat their acute migraines as they did prior to the study. Patients reported daily headache data using an electronic diary system, with compliance rates greater than $80 \%$. The analysis was performed using mixed-effects model reported measures (MMRM). A Migraine Disability Assessment (MIDAS) was administered to patients at baseline and after the 12-week study period to assess disability [107]. Compared with baseline, both doses of fremanezumab significantly decreased the number of migraine days relative to placebo in all three treatment cycles. Additionally, there was a greater reduction in the number of days of acute migraine medication use and in the number of headache days from baseline in the $225 \mathrm{mg}$ and $675 \mathrm{mg}$ fremanezumab groups relative to placebo, with no significant difference between the two doses. Patients on either dose of fremanezumab also demonstrated significant improvement from baseline in MIDAS scores relative to placebo. Subsequent post hoc analyses found that a larger percentage of the $225 \mathrm{mg}$ and $675 \mathrm{mg}$ groups experienced a $>50 \%$ reduction and $>75 \%$ reduction relative to the placebo group. The most common AEs reported were mild injection site reactions, which were similar across all three groups. No patients developed metabolic, immunologic, cardiac, or liver function abnormalities. There were no serious AEs considered to be related to fremanezumab treatment. Of note, $1 \%$ of patients $(n=2)$ had positive antibodies against fremanezumab prior to the study, and no subsequent antibody response was observed. In conclusion, this 12 -week trial demonstrated that both $225 \mathrm{mg}$ and $675 \mathrm{mg}$ of fremanezumab are effective in reducing the number of migraine days and acute medication use days, with a favorable safety profile.

A phase $2 \mathrm{~b}$ multicenter RCT was conducted to investigate the safety and efficacy of fremanezumab in the prevention of chronic migraine (CM) [81]. $\mathrm{CM}$ is defined as $\geq 15$ headache days per month, with $\geq 8$ migraine days per month for $>3$ months, per the ICHD-3 beta guidelines [1]. Patients in the US aged 18-65 and diagnosed with chronic migraines were eligible for the study. CM patients were allowed to be taking two preventive medications if they had been at the same dose for at least 3 months and to treat acute migraines as they did prior to study without restriction. Two dosing regimens were established: either an initial loading dose of $675 \mathrm{mg}$ followed by a monthly dose of $225 \mathrm{mg}$, or a consistent monthly dose of $900 \mathrm{mg}$. In all, 264 participants 
were randomized to $675 / 225 \mathrm{mg}$ fremanezumab, $900 \mathrm{mg}$ fremanezumab, or placebo for three 28-day treatment cycles, and they recorded daily headache data in an electronic diary. Analysis demonstrated a significantly greater reduction in the number of headache hours in both fremanezumab groups when compared with the placebo group during the third treatment cycle relative to baseline. Furthermore, there was a significantly larger reduction in the number of moderate to severe $(\mathrm{M} / \mathrm{S})$ headache days during the third treatment cycle in the fremanezumab groups relative to placebo. There was no significant difference in efficacy between either treatment group. A post hoc subgroup analysis demonstrated significantly fewer days per month of triptan use in the fremanezumab groups compared with placebo. Additionally, more patients in the $675 / 225 \mathrm{mg}$ group had $\mathrm{a} \geq 50 \%$ reduction in $\mathrm{M} / \mathrm{S}$ headache days than placebo, and more patients in the $900 \mathrm{mg}$ group had a $\geq 75 \%$ reduction in $\mathrm{M} / \mathrm{S}$ headache days than placebo. Similar to the phase 2b HFEM trial by Bigal et al. (2015a), the most common AEs across all groups were mild injection site reactions. There were no serious treatment-related AEs, and $1 \%$ of patients had antibodies against fremanezumab prior to the study and did not develop subsequent increases in antibody titers after taking fremanezumab. Both doses of fremanezumab demonstrated efficacy and safety in the preventive treatment of $\mathrm{CM}$.

Bigal et al. [108] conducted post hoc analyses of the previous study to assess the efficacy of fremanezumab at times points earlier than 3 months in CM patients [81, 82, 108]. A significant reduction in the number of headache hours from baseline was observed after 1 week of treatment with both doses of fremanezumab relative to placebo. A significant difference was first noted in the $900 \mathrm{mg}$ treatment arm by day 3 , and in the $675 / 225 \mathrm{mg}$ treatment arm by day 7. The percentage of patients with a 50\% reduction in the number of headache hours from baseline and in the number $\mathrm{M} / \mathrm{S}$ headache days increased from weeks 1 to 3 in both fremanezumab groups relative to placebo. Thus, both doses of fremanezumab showed superiority to placebo in the preventive treatment of
$\mathrm{CM}$, and some patients saw improvement within 1 week of treatment with fremanezumab. The rapid onset of relief provided by fremanezumab may increase treatment compliance among patients. However, it is not clear whether these results are clinically significant, as the patients were not interviewed at these early time points.

Cohen et al. [109] conducted pooled post hoc analyses of the data from Bigal et al. [81, 82] to evaluate the efficacy of fremanezumab as an add-on preventive therapy for patients with EM and $\mathrm{CM}$ on stable doses of standard migraine prevention medications [81, 82, 109]. They selected 133 patients with EM and CM who were taking preventive migraine medication at the time of study initiation and were randomized to either placebo $(n=66)$ or fremanezumab $(n=67)$. Efficacy endpoints were the number of migraine days, M/S headache days, and acute medication use days. Patients with add-on fremanezumab therapy had a significantly larger reduction in migraine days per month relative to placebo, with $50 \%$ or greater reduction seen in $40 \%$ of patients on fremanezumab and $24 \%$ of patients on placebo. There was also a significant decrease in the mean number of $\mathrm{M} / \mathrm{S}$ headache days in the fremanezumab group relative to placebo, with more patients on fremanezumab experiencing a $50 \%$ or greater decrease relative to placebo. Furthermore, fremanezumab significantly decreased the mean number of acute medication use days relative to placebo, with a larger percentage of patients experiencing a $50 \%$ or greater decrease relative to placebo. Ultimately, patients with $\mathrm{EM}$ and $\mathrm{CM}$ who were previously taking standard preventive migraine medications for at least 3 months saw a marked improvement with the addition of fremanezumab. Fremanezumab did not demonstrate interactions with major classes of preventive migraine medication and appeared to be safe when taken with other migraine medications. Lastly, decreasing acute migraine medication use can reduce the likelihood of headaches related to medication overuse [110].

Halker Singh et al. [111] subsequently performed a post hoc analysis of both trials by Bigal et al. $[81,82]$ to evaluate the sustained efficacy 
of fremanezumab during the full 3 months of treatment in EM and CM patients [81, 82, 111]. They found that a significantly greater percentage of HFEM patients on $225 \mathrm{mg}$ and $675 \mathrm{mg}$ fremanezumab sustained a 50\% reduction in migraine days, $\mathrm{M} / \mathrm{S}$ headache days, and days with acute medication use relative to placebo. Although there were few patients in any group who sustained a $75 \%$ or $100 \%$ reduction in these endpoints, a greater percentage was found in HFEM patients in either treatment arm relative to placebo. In the $\mathrm{CM}$ trial, although smaller differences were observed between the treatment arms and placebo, there was still a significantly higher percentage of patients taking fremanezumab with a sustained 50\% reduction in the study's endpoints. Again, there were more patients in the treatment cohorts who sustained $75 \%$ or $100 \%$ reduction in the endpoints relative to placebo, but the number of patients with these results were limited. Given the rising concerns about tachyphylaxis in migraine preventive therapy, this post hoc analysis demonstrates that fremanezumab has the potential to generate a sustained reduction in acute medication use, which may alleviate some of these concerns [111]. However, this analysis had multiple limitations, including small sample sizes, unknown types of acute migraine medication use, unknown quality-oflife measures, and a placebo response.

VanderPluym et al. [112] performed a post hoc analysis of the two phase $2 \mathrm{~b}$ RCTs conducted by Teva Pharmaceuticals described above [81, 82, 112]. Participants were asked whether they had a headache of any severity on that day, and questions assessing impairment in their physical performance, concentration, and mental fatigue. In the HFEM cohort, the treatment arms, regardless of dose, had a significant increase in headache-free days in which the patients were able to work, study, or do chores without difficulty and had no problems with concentration or prolonged time to task completion. In the $\mathrm{CM}$ arms, an impact was observed on the number of headache-free days where patients reported normal functioning (mental and physical), but it was seen primarily in the $900 \mathrm{mg}$ dose cohort as opposed to the $675 / 225 \mathrm{mg}$ dose cohort.
In an effort to quantify the time to response to fremanezumab, Silberstein et al. [113] performed post hoc analyses on the HFEM group described in Bigal et al. [81, 113]. All 297 participants from the study were included in the analyses. Investigators examined the drug response within the first 3 weeks of treatment versus the response in the placebo cohort. Within the first week, investigators found a significant decrease in all parameters measured in both treatment groups, including number of migraine days, number of $\mathrm{M} / \mathrm{S}$ headache days, number of headache hours, and number of days with nausea/vomiting, photophobia, or phonophobia. Migraine days decreased by 0.93 (0.49-1.36 fewer days, $p<0.0001)$ and 1.02 (0.58-1.46 fewer days, $p<0.0001)$ for the $225 \mathrm{mg}$ and $675 \mathrm{mg}$ treatment groups, respectively. This effect size remained stable, showing no increased efficacy after the first week. All other parameters continued to show a stable, significant reduction, apart from days with nausea/vomiting, which did not have a significant difference at week 3 in the $675 \mathrm{mg}$ treatment group. This analysis demonstrates the immediate preventive effect of fremanezumab with limited adverse effects. Additionally, it confirms a continued preventive effect in the weeks following the initial injection, which was maintained throughout the remainder of the study after injections of the same dose. The lack of dose titration and immediacy is a strength of fremanezumab when compared with currently approved preventive agents, which often require titration and have far more delayed preventive efficacy [114].

In a smaller phase 1 clinical trial conducted in 2016, Cohen-Barak et al. [115] reported results on the safety, tolerability, and pharmacokinetics of three doses of fremanezumab: $225 \mathrm{mg}, 675 \mathrm{mg}$, and $900 \mathrm{mg}$ [115]. Healthy Japanese and Caucasian participants $(n=32$ each) were randomized and equally distributed by ethnicity into one of four groups: placebo or a single dose of fremanezumab with $225 \mathrm{mg}$, $675 \mathrm{mg}$, or $900 \mathrm{mg}$. Patients were followed for 36 weeks after the administration of the study drug in an outpatient setting. There were a comparable number of AEs experienced by those in either treatment group when compared 
with placebo. The most common AE was injection site reaction. Moreover, no significant changes in clinical laboratory values or on electrocardiogram were observed. Of note, no patient developed antibodies to fremanezumab. Results from this phase 1 trial indicate that fremanezumab was well-tolerated in the short term at the doses studied, allowing for further studies at these doses.

Silberstein et al. [84] evaluated the efficacy and safety of fremanezumab for the prevention of $\mathrm{CM}$ in a randomized, double-blind cohort of 1130 individuals with $\mathrm{CM}$ per the International Classification of Headache Disorders, 3rd edition (ICHD-3), who were otherwise relatively healthy [1, 84]. Overall, 376 participants received fremanezumab monthly, 379 received the drug quarterly, and 375 received placebo. The dosing regimens were $675 \mathrm{mg}$ at baseline followed by placebo, or one $225 \mathrm{mg}$ injection at weeks 4 and 8 for the quarterly and monthly cohorts, respectively. The primary endpoint of the study was the average number of days with headache per month. Secondary endpoints included the change in the average number of days of migraine medication use per month, and reduction in the average number of migraine days per month and the six-item Headache Impact Test (HIT-6) score [116]. In comparison with placebo, both treatment cohorts demonstrated a statistically significant improvement in all endpoints, with no significant difference between the treatment groups. With respect to safety, there was no significant difference between placebo and the quarterly group. However, there were significantly more AEs reported in the monthly group (64\%-71\%, $P=0.03)$. Almost all AEs were reported to be of either mild or moderate severity (95-96\%), with injection site reactions constituting the majority of AEs among all groups. Notably, there was a $1 \%$ occurrence of transient elevated liver enzymes among all those receiving the trial drug. However, these participants were concurrently using agents known to potentially induce elevated liver enzymes. Additionally, there was no significant increase in this event when compared with the placebo cohort. In whole, this study suggests that fremanezumab may serve as an effective and safe agent in treating chronic migraines in relatively healthy persons.

Dodick et al. [83] compared fremanezumab with placebo for the prevention of episodic migraine (EM) in a cohort of 875 otherwise healthy participants, and patients in whom previous migraine medication management had failed were excluded [83]. EM was defined as "a headache occurring on 6-14 days, with at least 4 days fulfilling ICHD-3 criteria for migraine with aura (code 1.2; B and C) or without aura (code 1.1; C and D), probable migraine, or use of triptans or ergot derivatives" [1, 83]. Patients were once again randomized to monthly, quarterly, or placebo with 290, 291, and 294 participants in each group, respectively. Dosing for the treatment arms was an injection of $225 \mathrm{mg}$ at baseline with either a $225 \mathrm{mg}$ or placebo injection at weeks 4 and 8 for the monthly and quarterly groups, respectively. Investigators observed a significant change in the number of monthly migraine days in the 12 weeks following the baseline injection, the number of patients with a 50\% reduction in the number of monthly migraine days, and an improvement in the MIDAS score in both treatment groups [107]. Once again, there was no significant difference in efficacy between the two treatment cohorts. A greater number of treatment-related AEs were observed in the treatment groups; however, there was no difference in the number of serious AEs or AEs leading to discontinuation of the study. Injection site reactions were the most common AEs among all groups. There were also no clinically significant changes in objective data points, including liver function tests. Of note, four patients in the monthly group were positive for antibodies against fremanezumab, although there were no significant AEs in these patients. Overall, this study demonstrates fremanezumab's potential as preventive treatment of EM in patients who are otherwise healthy and have not had previous failure of preventive medications.

In the most recent post hoc analysis, Silberstein et al. [85] conducted a pooled analysis of safety and tolerability data from four phase $2 b$ and 3 trials on fremanezumab in the preventive treatment of migraine [81-85]. In total, 2566 
patients were randomized, and 2563 received either fremanezumab $(n=1704)$ or placebo $(n=86)$. The most common reasons for discontinuation of the study were withdrawal from study $(n=78)$, patient lost to follow-up $(n=60)$, and AEs $(n=50)$. Patients had an average age of 41 years and a 20-year history of diagnosed migraine. AEs were noted in 48-69\% of patients in all treatment groups, with the majority being mild reactions at the injection site. A total of $25-48 \%$ of patients reported one or more treatment-related AEs, with the majority being injection site reactions. Most AEs in the fremanezumab treatment groups that led to study discontinuation were mild to moderate and had resolved, and were most commonly injection site erythema and rash. Patients taking fremanezumab had significantly higher rates of AEs overall relative to placebo, with $48-69 \%$ of all treatment patients reporting $\geq 1$ AE $(p=0.001)$. Rates of AEs were similar between patients with concurrent preventive migraine medication use and those without. There were two deaths in the phase 3 trials that were determined to be unrelated to fremanezumab treatment. Fremanezumab did not appear to cause significant cardiovascular effects, with less than $1 \%$ of patients experiencing hypertension, tachycardia, or palpitations. Similarly, no significant evidence of liver toxicity was found, and most liver function abnormalities were mild to moderate and resolved without treatment. There were no cases of anaphylaxis or severe drug hypersensitivity. Although $0.4 \%$ of patients developed antidrug antibodies (ADA) after treatment with fremanezumab, there were no type I or III hypersensitivity AEs in those patients. Overall, this pooled analysis suggests that fremanezumab is safe and well-tolerated for the preventive treatment of migraine in patients with either EM or CM.

In 2019, Ferrari et al. [117] published data from their FOCUS study-a randomized, double-blind, placebo-controlled, parallel-group phase 3b study investigating fremanezumab's efficacy in hard-to-treat migraines [117]. Study participants were once again relatively healthy, although they could have minor comorbidities as determined by the investigator.
Notable participant criteria included that patients must have failed $2-4$ preventive treatments in the 10 years prior to screening and could not be taking preventive medications at the time of screening. EM and CM patients were included with similar criteria as previous studies; however, EM and CM participants required $\geq 4$ days and $\geq 8$ days, respectively, of headaches that met ICHD-3 criteria for migraine over the 28-day lead-in period [1]. Participants were randomized to either placebo $(n=279)$ or monthly $(n=283)$ or quarterly $(n=276)$ treatments for 12 weeks, followed by a 12 -week open-label period. All quarterly patients received $675 \mathrm{mg}$ at baseline, followed by placebo for the remainder of the blinded period. For the monthly EM cohort, dosing was $225 \mathrm{mg}$ per month, while the monthly $\mathrm{CM}$ cohort received $675 \mathrm{mg}$ at baseline, followed by 2 months of $225 \mathrm{mg}$. In the double-blinded period, all treatment cohorts demonstrated a significant decrease in the number of migraine days when compared with placebo. Moreover, the reduction was observed as early as the first month in both cohorts, as was a reduction in the average number of days triptans or ergots were used. Of note, $34 \%$ of participants in treatment groups had a $\geq 50 \%$ treatment response. With respect to patient-reported metrics, both HIT-6 and MIDAS scores were significantly improved compared with placebo $[107,116]$. Overall, there were no significant differences in efficacy between the study treatment groups or between EM and CM participants. Additionally, no significant difference in the incidence of AEs was observed. Injection site reactions were the most common $\mathrm{AE}$. Based on these findings, the FOCUS trial demonstrated that fremanezumab was efficacious and welltolerated for use in migraines unresponsive to traditional preventive treatments.

Despite current studies and these numerous analyses demonstrating that fremanezumab is relatively well-tolerated in the short term, concerns have been raised regarding its safety profile, and the safety profile of other CGRP ligand mAbs (eptinezumab, galcanezumab) and CGRP receptor mAbs (erenumab) [85, 118-120]. Some of this apprehension stems from CGRP's widereaching effects throughout the body, in 
addition to the fact that there is still uncertainty about the full extent of the role of CGRP in the body, despite significant research [121]. Majima et al. [118] note several potential adverse effects of long-term CGRP and CGRP receptor inhibitor use based on basic scientific research. Specific concerns relate to the inhibition of CGRP and its effects on vascularity. CGRP has been shown in multiple in vitro and mouse models to be pro-angiogenic, and to have pro-lymphangiogenic properties mediated by its action on macrophages and subsequent vascular endothelial growth factor (VEGF) secretion $[118,122,123]$. Mice studies have shown decreased wound healing and development of significant lymphedema when the CGRP gene was knocked out. It has also been suggested that CGRP mAbs could impact healing from ischemic injuries, such as stroke.

Moreover, CGRP has a profound impact on vascular resistance, serving as a potent vasodilator [118]. The inhibition of CGRP in its capacity as a vasodilator could foreseeably precipitate adverse effects. In a case report by Evans [120], three patients who were on CGRP or CGRP receptor antibody antagonists were reported to have Raynaud's phenomenon (RP) exacerbations or new onset [120]. Of note, patients with migraines are already at a significantly elevated risk of RP when compared with the general population [124]. One study, albeit small, showed that those with RP (from systemic sclerosis) had a reduced number of CGRP neurons in the skin by skin biopsy and immunohistochemistry [125]. Also, given CGRP's vasodilatory effects, it is conceivable that distal vasoconstriction, such as what is seen in $\mathrm{RP}$, is precipitated by CGRP antagonist use. Given the dearth of long-term follow-up studies and the small sample size of those who have taken fremanezumab, further studies and monitoring are needed before any definitive assessments can be made regarding the shortand long-term safety profile of fremanezumab.

Based on the aforementioned studies, Sacco et al. developed guidelines for the use of CGRP mAbs (eptinezumab, erenumab, fremanezumab, and galcanezumab) in migraine prevention, in accordance with the Grading of Recommendations, Assessment, Development and Evaluation (GRADE) system [126]. An expert panel of seven physicians evaluated 28 high-quality studies, including phase 2 or 3 RCTs, post hoc or pooled analyses of RCTs, and open-label trials, on the use of CGRP mAbs. In their evaluation of fremanezumab, the authors selected four RCTs investigating the safety and efficacy of fremanezumab relative to placebo in EM or CM patients, which were discussed in previous sections of this paper [81-84]. The panel developed the following expert consensus recommendations for the European Headache Federation on the use of CGRP mAbs in various clinical scenarios. They recommended CGRP mAbs in both EM and CM patients who failed two or more preventive treatments or who could not use other medications due to side effects or comorbidities. For EM patients, they suggested stopping preventive migraine medications before starting CGRP mAbs. For patients with $\mathrm{CM}$ already on a standard migraine preventive medication, they recommend adding a CGRP $\mathrm{mAb}$ and withdrawing the other drug at a later time to avoid rebound headaches. Similarly, the authors suggested adding a standard migraine prevention drug for $\mathrm{CM}$ patients already on a CGRP mAb and who might benefit from an additional medication. However, for $\mathrm{CM}$ patients on onabotulinumtoxinA with suboptimal response, discontinuance of the drug was recommended before starting a CGRP $\mathrm{mAb}$, as there is no current evidence of the efficacy or safety of combining those medications. The guidelines also suggest cessation of CGRP mAb use after 6-12 months of treatment in both EM and CM patients. Although a study on fremanezumab showed sustained efficacy in reducing migraine days during the full 3-month study period, there is insufficient data on the sustained benefit after 3 months or after withdrawal of CGRP mAbs. For migraines due to medication overuse in individuals with $\mathrm{CM}$, there is not enough information to suggest increased efficacy after withdrawal from the standard preventive migraine medication. Therefore, CGRP mAbs can be offered either before or after stopping the medication. Populations in whom use of CGRP mAbs is contraindicated include pregnant women, women who are breastfeeding, and patients with 
vascular disease (cardiovascular and cerebrovascular), drug or alcohol abuse, or severe mental disorders. CGRP is a strong vasodilator and is involved in blood pressure regulation, and therefore patients with cardiovascular and cerebrovascular disease were excluded from trials; thus there is insufficient data at this time to determine whether it would be safe in patients with vascular disease. However, the studies did not find a significant increase in cardiovascular events in patients taking CGRP mAbs. Lastly, multiple clinical trials have assessed for the development of binding or neutralizing antibodies against CGRP mAbs, and found no clinical significance associated with a positive result. Therefore, the guidelines suggest that current routine antibody testing in patients is not needed, but further studies with longer treatment duration are warranted before concluding that the development of these antibodies does not have clinical implications. With these clinical considerations, CGRP mAbs erenumab, fremanezumab, and galcanezumab have been demonstrated to be safe and effective for use in migraine prevention in selected patient populations.

\section{CONCLUSION}

Migraine headache is a common and debilitating neurovascular condition. The expression of CGRP in the trigeminovascular pain pathway plays a critical role in migraine pathogenesis [36, 77-79, 86-88]. Advances in therapies have made it possible to effectively prevent or reduce symptoms of migraine. Humanized monoclonal antibodies targeting CGRP and its receptor have recently been approved for migraine prophylaxis in adults $[75,79,91,101,127,128]$. Fremanezumab, a monoclonal antibody specific to CGRP, was approved by the FDA in September 2018 and by the EMA in March 2019 for migraine prevention in adults $[76,90]$. The safety, tolerability, and clinical efficacy of fremanezumab have been consistently validated across all placebo-controlled phase $2 \mathrm{~b} / 3$ trials in adults with episodic and chronic migraine [76, 77, 80-85]. Although currently available studies provide support for fremanezumab, further studies are needed to elucidate the relationship between the pharmacodynamic activity and its mechanism; the long-term risks, especially with regard to the cardiovascular system, of chronic use of anti-CGRP monoclonal antibodies; the specific sites of action, including whether it can bind to other human calcitonin receptors; and the effect of antidrug neutralizing antibodies on long-term efficacy [90, 129-133].

\section{ACKNOWLEDGEMENTS}

Funding. No funding or sponsorship was received for this study or publication of this article.

Authorship. All named authors meet the International Committee of Medical Journal Editors (ICMJE) criteria for authorship for this article, take responsibility for the integrity of the work as a whole, and have given their approval for this version to be published.

Disclosures. Ivan Urits, Gavin Clark, Daniel An, Bredan Wesp, Rebecca Zhou, Ariunzaya Amgalan, Amnon A. Berger, Hisham Kassem, Anh L. Ngo, Rachel J. Kaye, Elyse M. Cornett, and Omar Viswanath have nothing to disclose. Alan Kayes a member of the journal's Editorial Board.

Compliance with Ethics Guidelines. This article is based on previously conducted studies and does not contain any studies with human participants or animals performed by any of the authors.

Data Availability. Data sharing is not applicable to this article as no datasets were generated or analyzed during the current study.

Open Access. This article is licensed under a Creative Commons Attribution-NonCommercial 4.0 International License, which permits any non-commercial use, sharing, adaptation, distribution and reproduction in any medium or format, as long as you give appropriate credit 
to the original author(s) and the source, provide a link to the Creative Commons licence, and indicate if changes were made. The images or other third party material in this article are included in the article's Creative Commons licence, unless indicated otherwise in a credit line to the material. If material is not included in the article's Creative Commons licence and your intended use is not permitted by statutory regulation or exceeds the permitted use, you will need to obtain permission directly from the copyright holder. To view a copy of this licence, visit http://creativecommons.org/licenses/by$\mathrm{nc} / 4.0 /$.

\section{REFERENCES}

1. Headache Classification Committee of the International Headache Society (IHS). The international classification of headache disorders, 3rd edition. Cephalalgia. 2018;38(1):1-211. https://doi.org/10. 1177/0333102417738202.

2. Quintela E, Castillo J, Muñoz P, Pascual J. Premonitory and resolution symptoms in migraine: a prospective study in 100 unselected patients. Cephalalgia. 2006;26(9):1051-60.

3. Viana M, Sances G, Ghiotto N, Guaschino E, Allena M, Nappi G, Goadsby P, Tassorelli C. Variability of the characteristics of a migraine attack within patients. Cephalalgia. 2016;36(9):825-30.

4. GBD 2016 Headache Collaborators. Global, regional, and national burden of migraine and tensiontype headache, 1990-2016: a systematic analysis for the Global Burden of Disease study 2016. Lancet Neurol. 2018;17(11):954-76. https://doi.org/10. 1016/S1474-4422(18)30322-3.

5. Bigal ME, Serrano D, Buse D, Scher A, Stewart WF, Lipton RB. Acute migraine medications and evolution from episodic to chronic migraine: a longitudinal population-based study. Headache. 2008;48(8):1157-68.

6. GBD 2016 Disease and Injury Incidence and Prevalence Collaborators. Global, regional, and national incidence, prevalence, and years lived with disability for 328 diseases and injuries for 195 countries, 1990-2016: a systematic analysis for the Global Burden of Disease Study 2016. Lancet. 2017;390(10100):1211-59.
7. Buse DC, Loder EW, Gorman JA, Stewart WF, Reed ML, Fanning KM, Serrano D, Lipton RB. Sex differences in the prevalence, symptoms, and associated features of migraine, probable migraine and other severe headache: results of the American migraine prevalence and prevention (AMPP) study. Headache. 2013;53(8):1278-99.

8. Lipton R, Stewart W, Diamond S, Diamond M, Reed M. Prevalence and burden of migraine in the United States: data from the American Migraine Study II. Headache. 2001;41(7):646.

9. GBD 2015 DALYs and HALE Collaborators. Global, regional, and national disability-adjusted life-years (DALYs) for 315 diseases and injuries and healthy life expectancy (HALE), 1990-2015: a systematic analysis for the Global Burden of Disease Study 2015. Lancet. 2016;388(10053):1603-58.

10. GBD 2015 Neurological Disorders Collaborator Group. Global, regional, and national burden of neurological disorders during 1990-2015: a systematic analysis for the Global Burden of Disease Study 2015. Lancet Neurol. 2017;16(11):877-97.

11. Burch R, Rizzoli P, Loder E. The prevalence and impact of Migraine and severe headache in the United States: figures and trends from government health studies. Headache J Head Face Pain. 2018;58(4):496-505.

12. Burch RC, Loder S, Loder E, Smitherman TA. The prevalence and burden of migraine and severe headache in the United States: updated statistics from government health surveillance studies. Headache J Head Face Pain. 2015;55(1):21-34.

13. Stewart WF, Lipton RB, Celentano DD, Reed ML. Prevalence of migraine headache in the United States: relation to age, income, race, and other sociodemographic factors. JAMA J Am Med Assoc. 1992;267(1):64-9.

14. MacGregor EA, Hackshaw A. Prevalence of migraine on each day of the natural menstrual cycle. Neurology. 2004;63(2):351-3.

15. Ashina S, Serrano D, Lipton RB, Maizels M, Manack AN, Turkel CC, Reed ML, Buse DC. Depression and risk of transformation of episodic to chronic migraine. J Headache Pain. 2012;13(8):615-24.

16. Breslau N, Lipton RB, Stewart WF, Schultz LR, Welch KMA. Comorbidity of migraine and depression: investigating potential etiology and prognosis. Neurology. 2003;60(8):1308-12.

17. Bigal ME, Lipton RB. Modifiable risk factors for migraine progression. Headache J Head Face Pain. 2006;46(9):1334-43. 
18. Lipton RB, Serrano D, Nicholson RA, Buse DC, Runken MC, Reed ML. Impact of NSAID and triptan use on developing chronic migraine: results from the American Migraine Prevalence and Prevention (AMPP) study. Headache J Head Face Pain. 2013;53(10):1548-63.

19. Scher AI, Stewart WF, Lipton RB. Caffeine as a risk factor for chronic daily headache: a populationbased study. Neurology. 2004;63(11):2022-7.

20. Giffin NJ, Ruggiero L, Lipton RB, Silberstein SD, Tvedskov JF, Olesen J, Altman J, Goadsby PJ, Macrae A. Premonitory symptoms in migraine: an electronic diary study. Neurology. 2003;60(6):935-40.

21. Kelman L. The premonitory symptoms (prodrome): a tertiary care study of 893 migraineurs. Headache J Head Face Pain. 2004;44(9):865-72.

22. Becker WJ. The diagnosis and management of chronic migraine in primary care. Headache J Head Face Pain. 2017;57(9):1471-81.

23. Goadsby PJ, Lipton RB, Ferrari MD. Migraine-current understanding and treatment. $\mathrm{N}$ Engl J Med. 2002;346(4):257-70.

24. May A, Goadsby PJ. The trigeminovascular system in humans: pathophysiologic implications for primary headache syndromes of the neural influences on the cerebral circulation. Headache Q. $1999 ; 10(2): 155$.

25. Weiller C, May A, Limmroth V, Juptner M, Kaube H, Schayck RV, Coenen HH, Dlener HC. Brain stem activation in spontaneous human migraine attacks. Nat Med. 1995;1(July):658-60.

26. Akerman S, Holland PR, Goadsby PJ. Diencephalic and brainstem mechanisms in migraine. Nat Rev Neurosci. 2011;12(10):570-84.

27. Edvinsson L, Warfvinge K. Recognizing the role of CGRP and CGRP receptors in migraine and its treatment. Cephalalgia. 2019;39(3):366-73.

28. Charles A. The pathophysiology of migraine: implications for clinical management. Lancet Neurol. 2018;17(2):174-82.

29. Goadsby PJ, Holland PR, Martins-Oliveira M, Hoffmann J, Schankin C, Akerman S. Pathophysiology of migraine: a disorder of sensory processing. Physiol Rev. 2017;97(2):553-622.

30. Maniyar FH, Sprenger T, Monteith T, Schankin C, Goadsby PJ. Brain activations in the premonitory phase of nitroglycerin-triggered migraine attacks. Brain. 2014;137(1):232-41.
31. Schoonman GG, Evers DJ, Terwindt GM, Van Dijk JG, Ferrari MD. The prevalence of premonitory symptoms in migraine: a questionnaire study in 461 patients. Cephalalgia. 2006;26(10):1209-13.

32. Charles AC, Baca SM. Cortical spreading depression and migraine. Nat Rev Neurol. 2013;9(11):637-44.

33. Leao AAP. Spreading depression of activity in the cerebral cortex. J Neurophysiol. 1944;7(6):359-90.

34. Goadsby PJ (2012) Pathophysiology of migraine. Ann Indian Acad Neurol. 15(Suppl.):15-22.

35. Hadjikhani N, Sanchez Del Rio M, Wu O, Schwartz D, Bakker D, Fischl B, Kwong K, Cutrer F, Rosen B, Tootell R, Sorensen A, Moskowitz M. Mechanisms of migraine aura revealed by functional MRI in human visual cortex. Proc Natl Acad Sci USA. 2001;98(8):4687.

36. Paemeleire K, Maassenvandenbrink A. Calcitoningene-related peptide pathway $\mathrm{mAbs}$ and migraine prevention. Curr Opin Neurol. 2018;31(3):274-80.

37. Digre KB. Whats new in the treatment of migraine? J Neuro-Ophthalmology. 2019;39(3):352-9.

38. Eftekhari S, Salvatore CA, Johansson S, Chen TB, Zeng Z, Edvinsson L. Localization of CGRP, CGRP receptor, PACAP and glutamate in trigeminal ganglion. Relation to the blood-brain barrier. Brain Res. 2015;1600:93-109.

39. Tajti J, Uddman R, Möller S, Sundler F, Edvinsson L. Messenger molecules and receptor mRNA in the human trigeminal ganglion. J Auton Nerv Syst. 1999;76(2-3):176-83.

40. Hou M, Kanje M, Longmore J, Tajti J, Uddman R, Edvinsson L. 5-HT1B and 5-HT1D receptors in the human trigeminal ganglion: co-localization with calcitonin gene-related peptide, substance $\mathrm{P}$ and nitric oxide synthase. Brain Res. 2001;909(1-2): 112-20.

41. Dieterle A, Fischer MJ, Link AS, Neuhuber WL, Messlinger $\mathrm{K}$. Increase in CGRP-and nNOS-immunoreactive neurons in the rat trigeminal ganglion after infusion of an NO donor. Cephalalgia. 2011;31(1):31-42.

42. Burstein R, Noseda R, Borsook D. Migraine: multiple processes, complex pathophysiology. J Neurosci. 2015;35(17):6619-29.

43. Iyengar S, Ossipov MH, Johnson KW. The role of calcitonin gene-related peptide in peripheral and central pain mechanisms including migraine. Pain. 2017;158(4):543-59. 
44. Russo AF. CGRP: a new target for migraine. Annu Rev Pharmacol Toxicol. 2015;55(2):533-52.

45. Basbaum AI, Bautista DM, Scherrer G, Julius D. Cellular and molecular mechanisms of pain. Cell. 2009;139(2):267-84.

46. Yuan H, Spare NM, Silberstein SD. Targeting CGRP for the prevention of migraine and cluster headache: a narrative review. Headache. 2019;59(S2): 20-32.

47. Antonaci F, Ghiotto N, Wu S, Pucci E, Costa A. Recent advances in migraine therapy. Springerplus. 2016;5(1):1-14.

48. Cameron C, Kelly S, Hsieh SC, Murphy M, Chen L, Kotb A, Peterson J, Coyle D, Skidmore B, Gomes T, Clifford T, Wells G. Triptans in the acute treatment of migraine: a systematic review and network metaanalysis. Headache. 2015;55(S4):221-35.

49. Ong JJY, de Felice M. Migraine treatment: current acute medications and their potential mechanisms of action. Neurotherapeutics. 2018;15(2):274-90. https://doi.org/10.1007/s13311-017-0592-1.

50. Agostoni EC, Barbanti P, Calabresi P, Colombo B, Cortelli P, Frediani F, Geppetti P, Grazzi L, Leone M, Martelletti P, Pini LA, Prudenzano MP, Sarchielli P, Tedeschi G, Russo A. Current and emerging evidence-based treatment options in chronic migraine: a narrative review. J Headache Pain. 2019;20(1):92. https://doi.org/10.1186/s10194-019-1038-4.

51. Marmura MJ, Silberstein SD, Schwedt TJ. The acute treatment of migraine in adults: the American Headache Society evidence assessment of migraine pharmacotherapies. Headache. 2015;55(1):3-20.

52. Society AH. The American Headache Society position statement on integrating new migraine treatments into clinical practice. Headache. 2019;59(1): $1-18$.

53. Proietti Cecchini A, Grazzi L. Emerging therapies for chronic migraine. Curr Pain Headache Rep. 2014;18(4):408. https://doi.org/10.1007/s11916014-0408-5.

54. Ranoux D, Attal N, Morain F, Bouhassira D. Botulinum toxin type A induces direct analgesic effects in chronic neuropathic pain. Ann Neurol. 2008;64(3):274-83.

55. Mathew NT, Frishberg BM, Gawel M, Dimitrova R, Gibson J, Turkel C. Botulinum toxin type a (Botox) for prophylactic treatment of chronic daily headache: a randomized, double-blind, placebo-controlled trial. Headache. 2005;45(4):293-307.
56. Sandrini G, Perrotta A, Tassorelli C, Torelli P, Brighina F, Sances G, Nappi G. Botulinum toxin type- $\mathrm{a}$ in the prophylactic treatment of medication-overuse headache: a multicenter, double-blind, randomized, placebo-controlled, parallel group study. J Headache Pain. 2011;12(4):427-33.

57. Blumenfeld A, Silberstein SD, Dodick DW, Aurora SK, Turkel CC, Binder WJ. Method of injection of onabotulinumtoxina for chronic migraine: a safe, well-tolerated, and effective treatment paradigm based on the preempt clinical program. Headache. 2010;50(9):1406-18.

58. Barbanti P, Ferroni P. Onabotulinum toxin A in the treatment of chronic migraine: patient selection and special considerations. J Pain Res. 2017;10: 2319-29.

59. Aoki KR. Review of a proposed mechanism for the antinociceptive action of botulinum toxin type A. Neurotoxicology. 2005;26(5):785-93.

60. Aoki KR, Francis J. Updates on the antinociceptive mechanism hypothesis of botulinum toxin A. Park Relat Disord. 2011;17(SUPPL. 1):S28-33.

61. Naegel S, Obermann M. Topiramate in the prevention and treatment of migraine: efficacy, safety and patient preference. Neuropsychiatr Dis Treat. 2010;6(1):17-28.

62. Silberstein SD. Topiramate in migraine prevention: a 2016 perspective. Headache. 2017;57(1):165-78.

63. White HS. Molecular pharmacology of topiramate: managing seizures and preventing migraine. Headache. 2005;45(SUPPL. 1):48-56.

64. Schwedt TJ. Chronic migraine. Br Med J. 2014;348: g1416.

65. Do TP, Guo S, Ashina M. Therapeutic novelties in migraine: new drugs, new hope? J Headache Pain. 2019;20(1):1-13.

66. Taylor FR. CGRP, amylin, immunology, and headache medicine. Headache. 2019;59(1):131-50.

67. Chan C, Goadsby PJ. Recent advances in pharmacotherapy for episodic migraine. CNS Drugs. 2019;33(11):1053-71. https://doi.org/10.1007/ s40263-019-00665-9.

68. Dodick D, Lipton RB, Martin V, Papademetriou V, Rosamond W, VanDenBrink AM, Loutfi $\mathrm{H}$, Welch KM, Goadsby PJ, Hahn S, Hutchinson S, Matchar D, Silberstein S, Smith TR, Purdy RA, Saiers J. Consensus statement: cardiovascular safety profile of triptans (5-HT 1B/1D agonists) in the acute treatment of migraine. Headache. 2004;44(5):414-25. 
69. Puledda F, Messina R, Goadsby PJ. An update on migraine: current understanding and future directions. J Neurol. 2017;264(9):2031-9.

70. Negro A, Martelletti P. Gepants for the treatment of migraine. Expert Opin Investig Drugs. 2019;28(6): 555-67.

71. Tepper SJ. Anti-calcitonin gene-related peptide (CGRP) therapies: update on a previous review after the American headache society 60th scientific meeting, San Francisco, June 2018. Headache. 2018;58:276-90.

72. Ohlsson L, Kronvall E, Stratton J, Edvinsson L. Fremanezumab blocks CGRP induced dilatation in human cerebral, middle meningeal and abdominal arteries. J Headache Pain. 2018;19(1):66. https:// doi.org/10.1186/s10194-018-0905-8.

73. Edvinsson L. The CGRP pathway in migraine as a viable target for therapies. Headache. 2018;58: 33-47.

74. Bigal ME, Walter S, Rapoport AM. Therapeutic antibodies against CGRP or its receptor. Br J Clin Pharmacol. 2015;79(6):886-95.

75. Raffaelli B, Reuter U. The biology of monoclonal antibodies: focus on calcitonin gene-related peptide for prophylactic migraine therapy. Neurotherapeutics. 2018;15(2):324-35. https://doi.org/10.1007/ s13311-018-0622-7.

76. Teva Pharmaceuticals USA, Inc. Highlights for Prescribing Information. AJOVY TM (fremanezumabvfrm) injection, for subcutaneous use. Initial U.S. 2018. https://www.accessdata.fda.gov/drugsatfda docs/label/2018/761089s000lbl.pdf.

77. Hoy SM. Fremanezumab: first global approval. Drugs. 2018;78(17):1829-34.

78. Melo-Carrillo A, Noseda R, Nir R-R, Schain AJ, Stratton J, Strassman AM, Burstein R. Selective inhibition of trigeminovascular neurons by fremanezumab: a humanized monoclonal anti-CGRP antibody. J Neurosci. 2017;37(30):7149-63.

79. Edvinsson L, Haanes KA, Warfvinge K, Krause DN. CGRP as the target of new migraine therapies successful translation from bench to clinic. Nat Rev Neurol. 2018;14(6):338-50. https://doi.org/10. 1038/s41582-018-0003-1.

80. Silberstein SD, Cohen JM, Yeung PP. Fremanezumab for the preventive treatment of migraine. Expert Opin Biol Ther. 2019;19(8): 763-71.

81. Bigal ME, Edvinsson L, Rapoport AM, Lipton RB, Spierings ELH, Diener H-C, Burstein R, Loupe PS,
Ma Y, Yang R, Silberstein SD. Safety, tolerability, and efficacy of TEV-48125 for preventive treatment of chronic migraine: a multicentre, randomised, double-blind, placebo-controlled, phase $2 \mathrm{~b}$ study. Lancet Neurol. 2015;14(11):1091-100.

82. Bigal ME, Dodick DW, Rapoport AM, Silberstein SD, Ma Y, Yang R, Loupe PS, Burstein R, Newman LC, Lipton RB. Safety, tolerability, and efficacy of TEV48125 for preventive treatment of high-frequency episodic migraine: a multicentre, randomised, double-blind, placebo-controlled, phase $2 \mathrm{~b}$ study. Lancet Neurol. 2015;14(11):1081-90.

83. Dodick DW, Silberstein SD, Bigal ME, Yeung PP, Goadsby PJ, Blankenbiller T, Grozinski-Wolff M, Yang R, Ma Y, Aycardi E. Effect of fremanezumab compared with placebo for prevention of episodic migraine: a randomized clinical trial. JAMA. 2018;319(19):1999-2008.

84. Silberstein SD, Dodick DW, Bigal ME, Yeung PP, Goadsby PJ, Blankenbiller T, Grozinski-Wolff M, Yang R, Ma Y, Aycardi E. Fremanezumab for the preventive treatment of chronic migraine. $\mathrm{N}$ Engl J Med. 2017;377(22):2113-22.

85. Silberstein SD, McAllister P, Ning X, Faulhaber N, Lang N, Yeung P, Schiemann J, Aycardi E, Cohen JM, Janka L, Yang R. Safety and tolerability of fremanezumab for the prevention of migraine: a pooled analysis of phases $2 \mathrm{~b}$ and 3 clinical trials. Headache. 2019;59(6):880-90.

86. Maasumi K, Michael RL, Rapoport AM. CGRP and migraine: the role of blocking calcitonin gene-related peptide ligand and receptor in the management of migraine. Drugs. 2018;78(9):913-28.

87. Melo-Carrillo A, Strassman AM, Nir R-R, Schain AJ, Noseda R, Stratton J, Burstein R. Fremanezumab-a humanized monoclonal anti-cgrp antibody-inhibits thinly myelinated (A $\delta)$ but not unmyelinated (C) meningeal nociceptors. J Neurosci. 2017;37(44): 10587-96.

88. Moskowitz MA, Reinhard JF, Romero J, Melamed E, Pettibone DJ. Neurotransmitters and the fifth cranial nerve: is there a relation to the headache phase of migraine? Lancet. 1979;2(8148):883-5.

89. Goadsby PJ, Edvinsson L, Ekman R. Release of vasoactive peptides in the extracerebral circulation of humans and the cat during activation of the trigeminovascular system. Ann Neurol. 1988;23(2): 193-6.

90. Lionetto L, Curto M, Cisale GY, Capi M, Cipolla F, Guglielmetti M, Martelletti P. Fremanezumab for the preventive treatment of migraine in adults. Expert Rev Clin Pharmacol. 2019;12(8):741-8. 
91. Edvinsson L. The trigeminovascular pathway: role of CGRP and CGRP receptors in migraine. Headache. 2017;57(Suppl 2):47-55.

92. Edvinsson L. Functional role of perivascular peptides in the control of cerebral circulation. Trends Neurosci. 1985;8:126-31.

93. Goadsby P, Edvinsson L, Ekman R. Vasoactive peptide release in the extracerebral circulation of humans during migraine headache. Ann Neurol. 1990;28(2):183-7.

94. Ho TW, Edvinsson L, Goadsby PJ. CGRP and its receptors provide new insights into migraine pathophysiology. Nat Rev Neurosci. 2010;6:573-82.

95. Edvinsson L, Ekman R, Goadsby PJ. Measurement of vasoactive neuropeptides in biological materials: problems and pitfalls from 30 years of experience and novel future approaches. Cephalalgia. 2010;30(6):761-6.

96. Cernuda-Morollón E, Larrosa D, Ramón C, Vega J, Martínez-Camblor P, Pascual J. Interictal increase of CGRP levels in peripheral blood as a biomarker for chronic migraine. Neurology. 2013;81(14):1191-6.

97. Hansen JM, Hauge AW, Olesen J, Ashina M. Calcitonin gene-related peptide triggers migraine-like attacks in patients with migraine with aura. Cephalalgia. 2010;30(10):1179-86.

98. Lassen L, Haderslev P, Jacobsen V, Iversen H, Sperling $\mathrm{B}$, Olesen J. CGRP may play a causative role in migraine. Cephalagia. 2002;22(1):54-61.

99. Hansen JM, Ashina M. Calcitonin gene-related peptide and migraine with aura: a systematic review. Cephalalgia. 2014;34(9):695-707.

100. Mason RT, Peterfreund RA, Sawchenko PE, Corrigan AZ, Rivier JE, Vale WW. Release of the predicted calcitonin gene-related peptide from cultured rat trigeminal ganglion cells. Nature. 1984;308(5960): 653-5.

101. Ashina M, Hansen JM, Do TP, Melo-Carrillo A, Burstein R, Moskowitz MA. Migraine and the trigeminovascular system-40 years and counting. Lancet Neurol. 2019;18(8):795-804.

102. Eftekhari S, Salvatore CA, Calamari A, Kane SA, Tajti J, Edvinsson L. Differential distribution of calcitonin gene-related peptide and its receptor components in the human trigeminal ganglion. Neuroscience. 2010;169(2):683-96.

103. Miller S, Liu H, Warfvinge K, Shi L, Dovlatyan M, $\mathrm{Xu} \mathrm{C}$, Edvinsson L. Immunohistochemical localization of the calcitonin gene-related peptide binding site in the primate trigeminovascular system using functional antagonist antibodies. Neuroscience. 2016;328:165-83.

104. Lennerz JK, Rühle V, Ceppa EP, Neuhuber WL, Bunnett NW, Grady EF, Messlinger K. Calcitonin receptor-like receptor (CLR), receptor activity-modifying protein 1 (RAMP1), and calcitonin gene-related peptide (CGRP) immunoreactivity in the rat trigeminovascular system: differences between peripheral and central CGRP receptor distribution. J Comp Neurol. 2008;507(3):1277-99.

105. Eftekhari S, Warfvinge K, Blixt FW, Edvinsson L. Differentiation of nerve fibers storing CGRP and CGRP receptors in the peripheral trigeminovascular system. J Pain. 2013;14(11):1289-303.

106. Ning X, Cohen JM, Bennett NL, Yang R. Long-term safety of fremanezumab: results of a 1-year study. AAN Annual Meeting, Philadelphia, PA. 2019.

107. Stewart WF, Lipton RB, Kolodner KB, Sawyer J, Lee C, Liberman JN. Validity of the Migraine Disability Assessment (MIDAS) score in comparison to a diarybased measure in a population sample of migraine sufferers. Pain. 2000;88(1):41-52.

108. Bigal ME, Dodick DW, Krymchantowski AV, VanderPluym JH, Tepper SJ, Aycardi E, Loupe PS, Ma Y, Goadsby PJ. TEV-48125 for the preventive treatment of chronic migraine: efficacy at early time points. Neurology. 2016;87:41-8.

109. Cohen JM, Dodick DW, Yang R, Newman LC, Li T, Aycardi E, Bigal ME. Fremanezumab as add-on treatment for patients treated with other migraine preventive medicines. Headache. 2017;57(9): 1375-84.

110. Chiang C-C, Schwedt TJ, Wang S-J, Dodick DW. Treatment of medication-overuse headache: a systematic review. Cephalalgia. 2016;36(4):371-86.

111. Halker Singh RB, Aycardi E, Bigal ME, Loupe PS, McDonald M, Dodick DW. Sustained reductions in migraine days, moderate-to-severe headache days and days with acute medication use for HFEM and CM patients taking fremanezumab: post-hoc analyses from phase 2 trials. Cephalalgia. 2019;39(1): 52-60.

112. VanderPluym J, Dodick DW, Lipton RB, Ma Y, Loupe PS, Bigal ME. Fremanezumab for preventive treatment of migraine: functional status on headache-free days. Neurology. 2018;91(12):E1152-65.

113. Silberstein SD, Rapoport AM, Loupe PS, Aycardi E, McDonald M, Yang R, Bigal ME. The effect of beginning treatment with fremanezumab on headache and associated symptoms in the randomized phase 2 study of high frequency episodic migraine: 
post-hoc analyses on the first 3 weeks of treatment. Headache. 2019;59(3):383-93.

114. D'Amico D, Tepper SJ. Prophylaxis of migraine: general principles and patient acceptance. Neuropsychiatr Dis Treat. 2008;4:1155-67.

115. Cohen-Barak O, Weiss S, Rasamoelisolo M, Faulhaber N, Yeung PP, Loupe PS, Yoon E, Gandhi MD, Spiegelstein O, Aycardi E. A phase 1 study to assess the pharmacokinetics, safety, and tolerability of fremanezumab doses (225 mg, $675 \mathrm{mg}$ and $900 \mathrm{mg}$ ) in Japanese and Caucasian healthy subjects. Cephalalgia. 2018;38(13):1960-71.

116. Yang M, Rendas-Baum R, Varon SF, Kosinski M. Validation of the Headache Impact Test (HIT- ${ }^{\text {TM }}$ ) across episodic and chronic migraine. Cephalalgia. 2011;31(3):357-67.

117. Ferrari MD, Diener HC, Ning X, Galic M, Cohen JM, Yang R, Mueller M, Ahn AH, Schwartz YC, Grozinski-Wolff M, Janka L, Ashina M. Fremanezumab versus placebo for migraine prevention in patients with documented failure to up to four migraine preventive medication classes (FOCUS): a randomised, double-blind, placebo-controlled, phase 3b trial. Lancet. 2019;394(10203):1030-40.

118. Majima M, Ito Y, Hosono K, Amano H. CGRP/CGRP receptor antibodies: potential adverse effects due to blockade of neovascularization? Trends Pharmacol Sci. 2019;40(1):11-21.

119. Xu D, Chen D, Zhu LN, Tan G, Wang HJ, Zhang Y, Liu L. Safety and tolerability of calcitonin-gene-related peptide binding monoclonal antibodies for the prevention of episodic migraine - a meta-analysis of randomized controlled trials. Cephalalgia. 2019;39(9):1164-79.

120. Evans RW. Raynaud's phenomenon associated with calcitonin gene-related peptide monoclonal antibody antagonists. Headache. 2019;59(8):1360-4.

121. Russell FA, King R, Smillie SJ, Kodji X, Brain SD. Calcitonin gene-related peptide: physiology and pathophysiology. Physiol Rev. 2014;94:1099-142.

122. Toda M, Suzuki T, Hosono K, Hayashi I, Hashiba S, Onuma Y, Amano H, Kurihara Y, Kurihara H, Okamoto H, Hoka S, Majima M. Neuronal system-dependent facilitation of tumor angiogenesis and tumor growth by calcitonin gene-related peptide. Proc Natl Acad Sci USA. 2008;105(36):13550-5.

123. Kurashige C, Hosono $\mathrm{K}$, Matsuda $\mathrm{H}$, Tsujikawa $\mathrm{K}$, Okamoto H, Majima M. Roles ofreceptor activitymodifying protein 1 in angiogenesis and lymphangiogenesis during skin wound healing in mice. FASEB J. 2014;28(3):1237-47.

124. Zahavi I, Chagnac A, Hering R, Davidovich S, Kuritzky A. Prevalence of Raynaud's phenomenon in patients with migraine. Arch Intern Med. 1984;144(4):742-4.

125. Bunker CB, Terenghi G, Springall DR, Polak JM, Dowd PM. Deficiency of calcitonin gene-related peptide in Raynaud's phenomenon. Lancet. 2019;336(8730):1530-3.

126. Sacco S, Bendtsen L, Ashina M, Reuter U, Terwindt G, Mitsikostas D-D, Martelletti P. European headache federation guideline on the use of monoclonal antibodies acting on the calcitonin gene related peptide or its receptor for migraine prevention. J Headache Pain. 2019;20(1):6-39.

127. Bigal ME, Rapoport AM, Silberstein SD, Walter S, Hargreaves RJ, Aycardi E. From LBR-101 to fremanezumab for migraine. CNS Drugs. 2018;32(11): 1025-37.

128. Lionetto L, Curto M, Cisale GY, Capi M, Guglielmetti M, Martelletti P. Expert review of clinical pharmacology fremanezumab for the preventive treatment of migraine in adults. Expert. Rev Clin Pharmacol. 2019;12(8):741-8.

129. Favoni V, Giani L, Al-Hassany L, Asioli GM, Butera C, de Boer I, Guglielmetti M, Koniari C, Mavridis T, Vaikjärv M, Verhagen I, Verzina A, Zick B, Martelletti P, Sacco S, European Headache Federation School of Advanced Studies (EHF-SAS). CGRP and migraine from a cardiovascular point of view: what do we expect from blocking CGRP? J Headache Pain. 2019;20(1):27.

130. Deen M, Correnti E, Kamm K, Kelderman T, Papetti L, Rubio-Beltrán E, Vigneri S, Edvinsson L, Maassen Van Den Brink A. Blocking CGRP in migraine patients - a review of pros and cons. J Headache Pain. 2017;18:96.

131. Lionetto L, Cipolla F, Guglielmetti M, Martelletti P. Fremanezumab for the prevention of chronic and episodic migraine. Drugs Today (Barc). 2019;55(4): 265-76.

132. Bigal ME, Walter S, Rapoport AM. Fremanezumab as a preventive treatment for episodic and chronic migraine. Expert Rev Neurother. 2019;19(8): 719-28.

133. Raffaelli B, Neeb L, Reuter U. Monoclonal antibodies for the prevention of migraine. Expert Opin Biol Ther. 2019;19(12):1307-17. 